\title{
De la elegía del espacio a la elegía del territorio
}

\section{From the elegy of the space to the territorial's elegy}

\author{
Haesbaert, Rogério (2O I i), EL MITO DE LA DESTERRITORIALIZACIÓN: \\ DEL FIN DE LOS TERRITORIOS A LA MULTITERRITORIALIDAD, \\ SiglO XXI, MÉXICO, 328 PP., ISBN: 978-607-0308-I
}

Rogério Haesbaert en su libro El mito de la desterritorialización..., caracteriza el discurso de su obra complejo y ambiguo, incluso al interior de una misma perspectiva, como la que privilegia la dimensión económica de la sociedad (p.154).

Evidentemente que con la claridad y la destreza con la que él deshilvana las diferentes perspectivas que tratan el concepto, esta caracterización va mucho más allá de lo antes enunciado, pues en ocasiones parece que las diferentes posturas caen en la perturbación de algunos autores al tratar de interpretar una realidad cambiante o, bien, en la imprecisión de lo indecible que ocurre cuando quieren decir algo nuevo y diferente tratando de ganarle a la originalidad en la generación del conocimiento. Esta es una percepción parcial y particular sobre el tema que no tiene que ver con el escrito, sino con algunos temas integrados en su análisis.

Durante la lectura del libro me debatía con sentimientos también complejos que iban desde la alegría por contar con un texto de una claridad expositiva -a veces inusitada para demostrar las contradicciones y a veces las exageraciones de autores diversos para caracterizar el problemahasta la desesperación y angustia por llegar al final y ver cómo el autor concluía su exposición. A estas alturas, sin embargo, me encontraba apenas en el capítulo cinco, de nueve que componen el libro, en donde se desarrolla un tema importante para la geografía y para los interesados en los procesos territoriales, pues plantea una posible definición y categorización de la palabra territorio - muy usada en la actualidad- pero analizada a partir de su opuesto: la desterritorialización.

En el primer capítulo, el autor se centra en una interpretación sobre cómo el territorio y la desterritorialización son categorías descubiertas y estudiadas por las ciencias sociales, sobre todo en el último siglo.

En este sentido, comparto con el autor que no hay una definición clara de territorio, sin embargo, me parece que sólo la restringe a los deba- 
tes de la desterritorialización (p. 28), considero que incluso en la definición de territorio nos encontramos ante una categoría polisémica que se utiliza de maneras diversas por autores específicos; se advierte una disertación bastante bien documentada en el ámbito de la política y de la geografía en el capítulo dos del libro, el cual es complejo e impacta la detallada forma de examinar las diferentes visiones que existen sobre el concepto.

El capítulo tercero, en mi opinión, es todavía más complejo y en algunos casos hasta difícil de entender, pues ahí se expone la visión posestructuralista que desarrollan Deleuze y Guattari, autores que, al menos en el ámbito de la geografía mexicana, son bastante desconocidos y a quienes he visto referidos por algunos autores de esta corriente, en el ámbito de la geografía anglosajona como Massey (2005), Crang y Thrift, (2000) May y Thrift (2001), entre otros. Me parece que es de ellos de donde se toma la postura de la territorialización y desterritorialización de los procesos contemporáneos, con la cual tengo algunas diferencias que mencionaré más adelante.

En el capítulo cuarto se analiza la transición a una visión moderna y posmoderna del tema. En el capítulo cinco, mi ansiedad aumentaba, mientras se planteaba el mito de la desterritorialización, desarrollado en sus tres dimensiones: económica, política y cultural, anunciándose, sin embargo, un deslinde aparente del estructuralismo para definir las estructuras.

Especialmente interesante, por ser mi tema de trabajo, es el capítulo seis en donde se examina la desterritorialización y la movilidad para concluir con la discusión sobre las redes y la exclusión, tema tratado en el capítulo siete, y sobre la transición de la desterriotiralización a la multiterritorialización, planteada en el apartado ocho.

De este largo recorrido que tiene mucha hebra de donde cortar, tomaré sólo algunos temas para abordarlos explícitamente por limitaciones de tiempo y espacio.

El autor asume que se ha suscitado un debate con relación al tema; sin embargo, me pregunto ¿si con respecto a la desterritorialización ha habido realmente un debate, o se ha constituido una línea de pensamiento a partir de cómo diferentes autores la han definido o han asumido la categoría en sus discursos, y lo que es peor, lo han dado ya por un hecho? Creo más bien que no ha habido realmente una construcción sobre el tema y que el texto aquí presentado es un ícono con el que el debate se podría iniciar, al menos en los ámbitos de la geografía y la literatura social latinoamericana. En la visión de Perla Zusman, esta discusión da lugar a una de las formas en las que la geografía crítica se ha desarrollado en países como Brasil, a partir de las aportaciones que Rogério hace sobre este tema (Ramírez et al., 2013). 
Pero también es un texto que toma muchos aspectos de los debates contemporáneos, en donde llama la atención la disertación que sostienen diferentes autores sobre el tema cuando en ocasiones tienen estructuras del pensamiento bastante diferentes, y aún podríamos decir que hasta contradictorias; por ejemplo, se equiparan las ideologías de Harvey y Giddens, considerando a ambos como posmodernos (p. 135), siendo que uno es posmarxista, y el otro es moderno y hace una fuerte crítica al marxismo en alguno de sus textos. Asimismo, dependiendo de la referencia del autor que se tome, se pueden interpretar diferentes facetas de su propuesta sobre el territorio, o en este caso, del espacio. Es evidente que la manera de percibir a los autores es diferente, pues la forma como el autor percibió a Giddens, es bastante diversa de cómo lo vi yo, desde otra perspectiva (Ramírez, 2003).

Sin embargo, considero que hay al menos tres puntos controversiales que me gustaría poner sobre la mesa del debate, al menos en México: en primer lugar, me parece que en ocasiones hay un traslape en el uso de la categoría de espacio con la de territorio: casi todos los autores que hablan sobre territorio (aún en la económica, en la simbólica o cultural) tratan sobre cómo se eliminan las fronteras nacionales o se adecúa el Estado nación, o cómo culturalmente se produce una hibridación ante el inminente flujo de personas o mercancías, las culturas o los simbolismos que sobre ella se tienen, en donde el uso del territorio es sin duda claro, también lo hay cuando se habla de temas como control, aunque sea de agentes diversos y el Estado no aparezca. Sin embargo, algunos de los autores referidos en sus trabajos originales usan la definición de espacio, mientras que el autor confronta sus ideologías como si estuviésemos hablando de territorio (Massey, por ejemplo).

Me pregunto entonces si a pesar de este esfuerzo muy loable de aclaración y de redefinición que el autor hace, no existen imprecisiones que se presentan -tanto en la definición de territorio como en la de desterritorialización-, aunque hasta este momento he visto que el uso de esta categoría es más claro y preciso que el de la primera, ¿ No es acaso que está faltando la consideración de una dimensión hasta de diferencia lingüística, porque para las lenguas anglosajonas y francesa el hablar de territorio implica forzosamente la consideración de la dimensión estatal como factor fundamental para su consideración?, es decir, es para ellos una definición lingüística y no meramente académica. Esta diferenciación y necesidad de tomar en consideración los significados lingüísticos de los conceptos es algo que hemos argumentado en los últimos tiempos, ya que al no hacerse, genera confusiones originadas por la traducción a veces incorrecta del término y, que al no tomarlo en cuenta se hace una inter- 
pretación confusa y, en ocasiones, hasta errónea de las categorías usadas (López y Ramírez, 2012).

En algunos casos, el autor maneja indistintamente también las categorías de espacialidad y territorialidad (p.129) quedando poco claro en qué sentido se están usando, aunque a la segunda se le da un sentido claro de apropiación del territorio vivido.

La apreciación que he mencionado anteriormente, creo que se confirma cuando en el capítulo cuatro, el autor llega al tema de la posmodernidad y deja a un lado la dimensión política, adentrándose un poco en la discusión en torno a la redefinición de los procesos contemporáneos que tienen dos dimensiones: primero, es epistemológica y, por lo tanto, se ubica en la readecuación tiempo/espacio para definir el momento de tránsito; segundo, se ubica en el uso de la categoría de espacio, y no, en la de territorio. En un sentido, este aspecto que puede aparecer como liviano de la discusión, se muestra como un tránsito sin importancia; aunque desde mi punto de vista es de gran trascendencia, pues insisto, los conceptos no son similares, pero en ocasiones se sobreponen (p.125) y me parece que si a la discusión le agregamos los aportes que, sobre el tema, se han dado en el área del urbanismo marxista (Pradilla, 1984) y sociología urbana estructuralista (Coraggio, 1994), ambas latinoamericanas, el concepto de territorio parte de una crítica a la geografía espacialista y economicista neoclásica, argumentando la neutralidad del concepto y el uso de la categoría de territorio para asignar la vinculación naturaleza-sociedad en la apropiación, uso y transformación del territorio, en el primer caso, y de la configuración territorial, en caso de Coraggio, sin que necesariamente el control estatal político medie directamente para definirlo, sino que se integra de otro modo a la discusión.

En segundo lugar, el autor sugiere diferentes definiciones de territorio:

Teniendo como telón de fondo esta noción "híbrida" (y por tanto múltiple, nunca indiferenciada) del espacio geográfico, el territorio puede concebirse a partir de la imbricación de múltiples relaciones de poder, del poder material de las relaciones económico-políticas al poder simbólico de las relaciones de orden más estrictamente cultural (Haesbaert, 2011: 68).

Mientras que en la página 141 dice: "si concebimos al territorio en un sentido amplio de relaciones de poder, es factible afirmar que los objetivos o las razones de esta dominación y control (o descontrol en el caso de incluir la desterritorialización) pueden ser muy diversos y abarcar factores de orden económico, político y cultural”. Aquí está casi ausente la discusión estrictamente social del tema.

Sin embargo, en la página 194, dice que hay definiciones más integradoras, entre ellas se inclina por la que considera al territorio -o a los 
procesos de territorialización- "como fruto de la interacción entre las relaciones sociales y el control por el espacio, el cual implica relaciones de poder en sentido amplio, al mismo tiempo de manera más concreta (dominación) y más simbólica (un tipo de apropiación)".

Concluye en la página 281 con una definición en donde denomina a la territorialización como: "las relaciones de dominio y apropiación del espacio, o sea, la dimensión espacial de las relaciones de poder, en sentido amplio, desde el poder de efectos materiales más concretos hasta el más estrictamente simbólico". Por lo que me pregunto ¿cuál es la diferencia entre estas definiciones y cuáles son las más tradicionales que se encuentran sobre el tema? Desde mi perspectiva, son dos: una, que el dominio y control del que habla no necesariamente pasa por el que hace el Estado en la dimensión nacional u otras, sino que la abre a otras dimensiones y posibilidades con agentes que pueden integrarse de otras maneras; dos, que se incluye la posibilidad de entender al territorio como una dimensión procesual que integra a la desterritorialización como parte de la transformación que sufre en su devenir en el tiempo en donde la reterritorialización es parte también de su proceso. Es evidente que toman una postura posestructuralista al afirmar que asume y resalta "el aspecto temporal, dinámica y en red que el territorio también asume... en que la 'integración' de sus múltiples dimensiones es vista a través de las relaciones conjuntas de dominación y apropiación, o sea, de relaciones de poder en sentido amplio" (p. 281).

Es aquí en donde, a mi entender, el carácter del proceso se integra de una manera muy fragmentada, característica del estructuralismo, en donde las partes que componen el proceso se desintegran, para posteriormente integrarse parcialmente en un devenir alternado de lo que se controla o se apropia, luego se descontrola o desapropia (desterritorialización) para volverse a recontrolar o reapropiar. Es en esta disociación en la que parecería que al territorializar se está hablando de un tipo de espacio específico - ¿fijo se podría llamar?- que sería diferente al que tiene la desterritorialización, en donde la noción de espacio de flujos y la movilidad se integran para luego reterritorializarse y volver a hacerse fijo, utilizando conceptos de Castells y de Santos, aceptando el concepto de espacio de fijos y flujos de Milton Santos, en su momento. De esta manera estamos ante un proceso que tiene un soporte territorial cuando es territorio, se convierte en espacio y en flujo cuando se desterritorializa, pero me pregunto ¿sin base material?, y para posteriormente regresar a su estado territorial cuando se reterritorializa; sin embargo, las mediaciones que se requieren para entender estos pasos no están construidas ni explicadas en el desarrollo que se hace sobre el tema. 
Si tomamos en cuenta, una vez más, el aporte que algunos urbanistas marxistas han hecho al proceso territorial basados en un concepto de territorio como fundamento para la circulación diferencial y fragmentada, más que como espacio de flujos, la aparente deslocalización adquiere continuidad a partir de un conjunto de soportes materiales que articulan el territorio inicial (sea este económico, social, político o simbólico) con el de la reterritorialización.

Desde esta perspectiva, si bien existe un cambio de localización con la movilidad, la deslocalización no existe, pues puede ser considerada como un tránsito entre territorios, que para hacerlo, cuentan con un soporte territorial. Aun las transacciones electrónicas cuentan con la simultaneidad del tiempo real, poseen soportes que lo permiten en ambos lugares. Desde este punto de vista, se deslocaliza, pero no se desterritorializa, pues siempre tiene soporte con una base material que le permite su reproducción y con quien tiene relación con su tierra, con su naturaleza o con los agentes con los que se encuentra, pero a su vez que le permite relacionarse con otros agentes.

En suma, si bien resuelve las disociaciones de las estructuras política, económica y cultural del estructuralismo, al tomar las posturas post de Deleuze y Guatari conjunta, a veces, con la de Massey que los retoma del todo, desde mi lectura regresa a otro tipo de disociación del proceso que impide ver la manera como se usan las mercancías, los agentes o los símbolos en las diferentes fases de la circulación de la naturaleza, generando formas territoriales específicas y diversas que se articulan en un proceso conjunto que se territorializa continuamente en diferentes soportes, pero siempre territorializados.

El tercer punto refiere a la manera como el autor analiza la coexistencia entre el territorio con su dimensión zonal, las redes que se conforman a partir del espacio de flujos y los aglomerados de exclusión para explicar la multiterritorialidad, concepto que me parece bastante innovador, pero que al construirse en su conjunto, estimo que adolece una vez más de la fragmentación de los elementos del proceso que es parte de la visión estructuralista. Si bien los dos primeros, territorios y redes son definidos y caracterizados ampliamente y de manera dicotómica (véase cuadro 7.2 de la página 239), los aglomerados de exclusión, en tanto que resultado de un proceso, más que representar zonas en sí mismas, son resultado de condiciones sociales precarizadas pero que

... en la construcción de territorios 'bajo control' (término redundante) o 'autónomos' se vuelve... subordinada a intereses ajenos a la población que allí se reproduce. El aparente desorden que rige esta condición, en el sentido negativo desorden, es fruto de la no identificación de los grupos 
con su ambiente y la ausencia de control del espacio por sus principales 'usuarios'. Evidentemente que los trabaja como desterritorialización pero en donde los excluidos intentan en todo momento afirmarse, es decir, reterritorializarse.

Esta visión de tres áreas en la multiterritorialidad que parecería que siguen procesos autónomos que no tienen que ver unos con otros y que hasta persiguen trayectorias autónomas me remiten a la discusión que se dio en América Latina sobre los marginados en la década de los años setenta del siglo pasado, cuando se les consideró independientes del capitalismo imperante en el momento, por lo que podían entre ellos reproducirse en las áreas marginales de la ciudad que no tenían nada que ver con las desarrolladas. Esta aparente independencia entre los territorios parecería que no es fruto de procesos articulados que de alguna manera se vieron o conjuntaron elementos que los vinculan. Otras zonas como los basureros o las zonas de residuos nucleares, las Áreas Naturales Protegidas y otras, son el resultado de la manera como se percibe el carácter de un territorio en el sistema de control dominante. Con respecto a este tema también me parece que la visión estructuralista en lugar de integrar la visión, como es el objetivo del autor, la desarticula y la hace aparecer como independiente del proceso de desarrollo integral del territorio.

Sobre esta lectura quedan pendientes muchos temas por platicar con el autor, entre ellos el de las escalas, la vinculación entre la discusión epistemológica espacio/tiempo y el territorio; entre otros. Sin embargo sí me gustaría comentar que me agradó encontrarme con un texto que, entre otras cosas, ha tomado el giro cultural que las ciencias sociales han adoptado en el momento contemporáneo sin que medie un sobredeterminismo cultural que se percibe en otros libros, además de que tiene un peso relevante pero equilibrado en relación con las otras dimensiones de las estructuras, como son la económica y la política.

Agradezco al autor haber escrito este libro que ha sido un verdadero regalo para mi espíritu crítico en el ámbito de la geografía, en donde mi insistencia por hacer una ciencia diferente, de carácter teórico y político se inserta perfectamente en las discusiones y aportes que esta obra me proporciona.

\section{Bibliografía}

Coraggio, José Luis, (1994 [1987]), Territorios en transición. Crítica a la planificación regional en América Latina, Universidad Autónoma del Estado de México, México. 
Crang, Mike y Nigel Thrift (2000), Thinking space, Routledge, London.

López Liliana y Blanca Ramírez (2012), "Pensar el espacio: región, paisaje, territorio y lugar en las ciencias sociales", en Ma. Eugenia Reyes Ramos y Álvaro López Lara, Explorando Territorios. Una visión desde las ciencias sociales, Portafolios de Sociología núm. 2, Universidad Autónoma Metropolitana Xochimilco, México.

Massey, Doreen (2005), For space, Sage, London.

May, Jon y Nigel Thrift, (2001), Timespace, geographies of temporality, London, Routledge.

Pradilla, Emilio (1984), Contribución a la crítica de la "teoría urbana" del "espacio" a la "crisis urbana", Universidad Autónoma Metropolitana Xochimilco, México.

Ramírez, Blanca (2003), Modernidad, posmodernidad, globalización y territorio: un recorrido por los campos de las teorías, Miguel Ángel Porrúa-Universidad Autónoma Metropolitana Xochimilco, México.

Ramírez, Blanca, Gustavo Montañez y Perla Zusman (2013), "Geografías críticas latinoamericanas", en Martha Chávez y Martín Checa (eds.), en proceso de publicación por el Colegio de Michoacán.

Recibido: 05 de febrero de 2013. Aceptado: 15 de febrero de 2013.

Blanca Rebeca Ramírez Velázquez Universidad Autónoma Metropolitana, Unidad Xochimilco Correo-e: blare19@prodigy.net.mx

Blanca Rebeca Ramírez Velázquez. Es doctora en urbanismo por el posgrado de la Universidad Nacional Autónoma de México, adscrita al Departamento de Teoría y Análisis de la Universidad Autónoma Metropolitana Xochimilco. Profesora del posgrado en Ciencias y Artes para el Diseño, en Ciencias Sociales de la UAM, así como en el posgrado de Urbanismo en la UNAM. Es miembro del Sistema Nacional de Investigadores, nivel II. Sus líneas de investigación son: Globalización y transformaciones territoriales y de movilidad laboral en la región centro de México. Entre 
sus últimas publicaciones destacan, en coautoría: "Movilidad laboral en la construcción de la ciudad región del centro del país. Dispersión y encuentro de procesos", en Pradilla, Emilio (comp.), Ciudades compactas, dispersas, fragmentadas, UAM-Miguel Ángel Porrúa, México, pp. 295-325 (2011); "Espacio y política en el desarrollo territorial”, en Economía, Sociedad y Territorio, vol. XI, núm. 37, septiembre-diciembre, El Colegio Mexiquense, A.C., Zinacantepec, pp. 553-574, (2011); en coautoría, "Pensar el espacio: la región, el paisaje, el territorio y el lugar en las ciencias sociales”, en María Eugenia Reyes y Álvaro López (comps.), Explorando territorios: una visión desde las ciencias sociales, División de Ciencias Sociales, colección Portafolios de Sociología núm 2, México (2012); "La vulnerabilidad territorial del neoliberalismo mexicano", en Calva, José Luis (coord.), Desarrollo regional y urbano, análisis estratégico para el desarrollo, vol. 13, Consejo Nacional de Universitarios, Juan Pablos editores, México, pp. 251-272, (2012). 